\title{
Genome-wide association analyses identify variants in IRF4 associated with acute myeloid leukemia and myelodysplastic syndrome susceptibility
}

Junke Wang ${ }^{1 ¥}$, Alyssa I. Clay-Gilmour ${ }^{2}$, Ezgi Karaesmen ${ }^{1}$, Abbas Rizvi ${ }^{1}$, Qianqian Zhu ${ }^{3}$, Li Yann, Leah Preus ${ }^{1}$, Song Liu ${ }^{3}$, Yiwen Wang ${ }^{1}$, Elizabeth Griffiths ${ }^{4}$, Daniel O. Stram ${ }^{5}$, Loreall Pooler ${ }^{5}$, Xin Sheng ${ }^{5}$, Christopher Haimann, David Van Den Berg ${ }^{5}$, Amy Webb 6 , Guy Brock ${ }^{6}$, Stephen Spellman ${ }^{7}$, Marcelo Pasquini ${ }^{8}$, Philip McCarthy ${ }^{9}$, James Allan ${ }^{10}$, Friedrich Stölzel ${ }^{11}$, Kenan Onel ${ }^{12}$, Theresa Hahn ${ }^{4 *}$, Lara E. Sucheston-Campbell ${ }^{1,13 *}$

${ }^{1}$ College of Pharmacy, The Ohio State University

${ }^{2}$ Department of Epidemiology, Mayo Clinic

${ }^{3}$ Department of Biostatistics and Bioinformatics, Roswell Park Comprehensive Cancer Center

${ }^{4}$ Department of Medicine, Roswell Park Comprehensive Cancer Center

${ }^{5}$ Department of Preventive Medicine, University of Southern California

${ }^{6}$ Department on Biomedical Informatics, The Ohio State University

${ }^{7}$ Center for International Blood and Marrow Transplant Research, Minneapolis Campus

${ }^{8}$ Center for International Blood and Marrow Transplant Research, Medical College of Wisconsin

${ }^{9}$ Department of Medicine, Roswell Park Comprehensive Cancer Center

${ }^{10}$ Northern Institute for Cancer Research, Newcastle University, UK

${ }^{11}$ Department of Internal Medicine I, University Hospital Carl Gustav Carus Dresden, Technical University Dresden,

${ }^{12}$ Department of Pediatrics, Mount Sinai

${ }^{13}$ College of Veterinary Medicine, The Ohio State University

* Joint first and joint last authors

Running head: GWAS in AML and MDS

Keywords: acute myeloid leukemia, myelodysplastic syndrome, genome-wide association study, blood and marrow transplantation, pleiotropy

Word Count: 3754

\section{Conflicts of interest: None}

\section{Corresponding author:}

Lara E. Sucheston-Campbell, MS, PhD, The Ohio State University, 496 W. 12th Ave., 604 Riffe Building, Columbus, OH 43210; email: sucheston-campbell.1@osu.edu; phone: 614-688-2502 


\section{ABSTRACT}

2 The role of common genetic variation in susceptibility to acute myeloid leukemia (AML),

3 and myelodysplastic syndrome (MDS), a group of rare clonal hematologic disorders

4 characterized by dysplastic hematopoiesis and high mortality, remains unclear. We

5 performed AML and MDS genome-wide association studies (GWAS) in the DISCOVeRY-

6 BMT cohorts (2309 cases and 2814 controls). Association analysis based on subsets

7 (ASSET) was used to conduct a summary statistics SNP-based analysis of MDS and

8 AML subtypes. For each AML and MDS case and control we used PrediXcan to estimate

9 the component of gene expression determined by their genetic profile and correlate this

10 imputed gene expression level with risk of developing disease in a transcriptome-wide

11 association study (TWAS). ASSET identified an increased risk for de novo AML and MDS

$12\left(\mathrm{OR}=1.38,95 \% \mathrm{Cl}, 1.26-1.51, \mathrm{P}_{\text {meta }}=2.8 \times 10^{-12}\right)$ in patients carrying the $\mathrm{T}$ allele at

13 rs12203592 in Interferon Regulatory Factor 4 (IRF4), a transcription factor which

14 regulates myeloid and lymphoid hematopoietic differentiation. Our TWAS analyses

15 showed increased IRF4 gene expression is associated with increased risk of de novo

16 AML and MDS $\left(\mathrm{OR}=3.90,95 \% \mathrm{Cl}, 2.36-6.44, \mathrm{P}_{\text {meta }}=1.0 \times 10^{-7}\right)$. The identification of IRF4

17 by both GWAS and TWAS contributes valuable insight on the role of genetic variation in

$18 \quad \mathrm{AML}$ and MDS susceptibility. 


\section{INTRODUCTION}

22 Genome-wide association studies (GWAS) have been successful at identifying risk loci

23 in several hematologic malignancies, including acute myeloid leukemia (AML) ${ }^{1-3}$.

24 Recently genomic studies have identified common susceptibility loci between chronic

25 lymphocytic leukemia (CLL), Hodgkin lymphoma (HL), and multiple myeloma 26 demonstrating shared genetic etiology between these B-cell malignancies (BCM) ${ }^{4-6}$.

27 Given the evidence of a shared genetic basis across BCM and the underlying genetic 28 predisposition for AML and myelodysplastic syndromes (MDS) observed in family, 29 epidemiological, and genetic association studies ${ }^{1,7-9}$, we hypothesized that germline

30 variants may contribute to both AML and MDS development. Using the DISCOVeRY31 BMT study population (2309 cases and 2814 controls), we performed AML and MDS

32 GWAS in European Americans and used these data sets to inform our hypothesis. To

33 address the disease heterogeneity within and across our data we used a validated meta-

34 analytic association test based on subsets (ASSET) ${ }^{4}$. ASSET tests the association of

35 SNPs with all possible AML and MDS subtypes and identifies the strongest genetic association signal. To systematically test the association of genetically predicted gene expression with disease risk, we performed a transcriptome wide association study 38 (TWAS) ${ }^{10,11}$. This allows a preliminary investigation into the role of non-coding risk loci, which might be regulatory in nature, that impact expression of nearby genes. The TWAS

40 statistical approach, PrediXcan ${ }^{11}$, was used to impute tissue-specific gene expression

41 from a publicly available whole blood transcriptome panel into our AML and MDS cases

42 and controls. The predicted gene expression levels were then tested for association with

43 AML and MDS. The use of both a GWAS and TWAS in the DISCOVeRY-BMT study 
44 population allowed us to identify $A M L$ and MDS associations with IRF4, a transcription

45 factor which regulates myeloid and lymphoid hematopoietic differentiation, and has been

46 previously identified in GWAS of BCM.(5)

\section{MATERIALS AND METHODS}

Study Design \& Population

51 Our study was a nested case-control design derived from the parent study DISCOVeRY-

52 BMT (Determining the Influence of Susceptbility COnveying Variants Related to 1-Year

53 Mortality after unrelated donor Blood and Marrow Transplant). ${ }^{12}$ The DISCOVeRY-BMT

54 cohort was compiled from 151 centers around the world through the Center for

55 International Blood and Marrow Transplant Research (CIBMTR). Briefly, the parent study

56 was designed to find common and rare germline genetic variation associated with survival

57 after an URD-BMT. DISCOVeRY-BMT consists of two cohorts of ALL, AML and MDS

58 patients and their 10/10 human leukocyte antigen (HLA)-matched unrelated healthy

59 donors. Cohort 1 was collected between 2000 and 2008, Cohort 2 was collected from

$60 \quad 2009-2011$.

61

62 AML and MDS patients were selected from the DISCOVeRY-BMT patient cohorts and

63 used as cases and all the unrelated donors from both cohorts as controls. AML subtypes

64 included de novo AML with normal cytogenetics, de novo AML with abnormal 65 cytogenetics and therapy-related AML (t-AML). De novo AML patients did not have 66 precedent MDS, chemotherapy or radiation for prior cancers. MDS subtypes included de 
novo MDS, defined as patients without precedent chemotherapy or radiation for prior cancers, and therapy-related MDS (t-MDS). Patient cytogenetic subtypes were available, however due to limited sample sizes for each cytogenetic risk group, we consider here only broad categories. Controls were unrelated, healthy donors aged 18-61 years who passed a comprehensive medical exam and were disease-free at the time of donation. All patients and donors provided written informed consent for their clinical data to be used for research purposes and were not compensated for their participation.

\section{Genotyping, imputation, and quality control}

Genotyping and quality control in the DISCOVeRY-BMT cohort has previously been described in detail ${ }^{12-15}$. Briefly, samples were assigned to plates to ensure an even distribution of patient characteristics and genotyping was performed at the University of Southern California Genomics Facility using the Illumina Omni-Express BeadChip® containing approximately 733,000 single nucleotide polymorphisms (SNPs). ${ }^{16}$ SNPs were

81 removed if the missing rate was $>2.0 \%$, minor allele frequency $(\mathrm{MAF})<1 \%$, or for violation of Hardy Weinberg equilibrium proportions $\left(P<1.0 \times 10^{-4}\right)$.

84 Problematic samples were removed based on the SNP missing rate, reported-genotyped sex mismatch, abnormal heterozygosity, cryptic relatedness, and population outliers.

86 Population stratification was assessed via principal components analysis using Eigenstrat software $^{17}$ and a genomic inflation factor $(\lambda)$ was calculated for each cohort. Following SNP quality control, 637,655 and 632,823 SNPs from the OmniExpress BeadChip in

89 Cohorts 1 and 2, respectively were available for imputation. SNP imputation was 
90 performed using Haplotype Reference Consortium, hg19/build 37 (http://www.haplotype-

91 reference-consortium.org/home) via the Michigan Imputation server ${ }^{18,19}$. Variants with

92 imputation quality scores $<0.8$ and minor allele frequency $(\mathrm{MAF})<0.005$ were removed

93 yielding almost 9 million high quality SNPs available for analysis in each cohort.

\section{METHODS}

96 Statistical Analysis

97 Genome-wide SNP associations with AML and MDS

98 Quality control and statistical analyses were implemented using QCTOOL-v2, R 3.5.2

99 (Eggshell Igloo), Plink-v1.9, and SNPTEST-v2.5.4-beta3. Logistic regression models 100 adjusted for age, sex, and three principal components were used to perform single SNP 101 tests of association with de novo MDS, t-MDS, AML by subtype (de novo AML with normal 102 cytogenetics, de novo AML with abnormal cytogenetics and t-AML) in each cohort. 103 European American healthy donors were used as controls. SNP meta-analyses of 104 cohorts 1 and 2 were performed by fitting random effects models. ${ }^{20}$ To identify the 105 strongest association signal with AML and MDS we conducted a summary statistic SNP106 based association analysis (ASSET) implemented in R statistical software ${ }^{4}$. ASSET tests 107 each SNP for association with outcome using an exhaustive search across non108 overlapping AML and MDS case groups while accounting for the multiple tests required 109 by the subset search, as well as any shared controls between groups ${ }^{4}$. 
112 We calculated heritability of AML and MDS combined and by independent subtypes as

113 the proportion of phenotypic variance explained by all common genotyped SNPs, using

114 the genome-based restricted maximum likelihood method performed with the Genome-

115 wide Complex Trait Analysis (GCTA) software. ${ }^{21-23}$ We report heritability on the observed

116 scale due to genome-wide genotyped variants as well as heritability on the liability scale

117 assuming AML and MDS disease prevalence of $0.0001 .{ }^{24-26}$

119 Transcriptome-wide association study (TWAS) of AML and MDS

120 To prioritize GWAS findings and identify expression quantitative trait loci (eQTL)-linked

121 genes, we carried out a gene expression tests of association of de novo AML and MDS

122 using PrediXcan ${ }^{11}$. This method leverages the well-described functional regulatory

123 enrichment in genetic variants relatively close to the gene body (i.e. cis-regulatory

124 variation) to inform models relating SNPs to gene expression levels in data with both gene

125 expression and SNP genotypes available. Robust prediction models are then used to

126 estimate the effect of cis-regulatory variation on gene expression levels. Using imputation,

127 the cis-regulatory effects on gene expression from these models can be predicted in any

128 study with genotype measurements, even if measured gene expression is not available.

129 Thus, we imputed the cis-regulatory component of gene expression into our data for each

130 individual using models trained on the whole blood transcriptome panel $(n=922)$ from the

131 Depression Genes and Networks (DGN) ${ }^{27}$, yielding expression levels of 11,200 genes for

132 each case and control. The resulting estimated gene expression levels were then used

133 to perform gene-based tests of differential expression between AML and MDS cases and 134 controls adjusted for age and sex. A fixed effects model with inverse variance weighting 
135 using the $\mathrm{R}$ package Metafor was used for meta-analysis of cohorts 1 and 2 . A

136 Bonferroni-corrected transcriptome wide significance threshold was set at $\mathrm{P}<4.5 \times 10^{-6}$.

138 Functional Annotation of Genetic Variation associated with AML and MDS

139 To better understand the potential function of the variants identified by GWAS and ASSET 140 analyses we annotated significant SNPs using publicly available data. eQTLGen, a

141 consortium analyses of the relationship of SNPs to gene expression in 30,912 whole

142 blood samples, was used to determine if significant and suggestive SNPs $\left(p<5 \times 10^{-6}\right)$

143 were whole blood cis-eQTL, defined as allele specific association with gene expression

$144 \quad 28$. Genotype-Tissue Expression project (GTEx) was used to test for significant eQTLs in $145>70$ additional tissues ${ }^{29}$. AML and MDS SNP associations were also placed in context of 146 previous GWAS using Phenoscanner, a variant-phenotype comprehensive database of 147 large GWAS, which includes results from the NHGRI-EBI GWAS catalogue, the UK 148 Biobank, NIH Genome-Wide Repository of Associations between SNPs and Phenotypes 149 and publicly available summary statistics from more than 150 published genome 150 association studies. Results were filtered at $\mathrm{P}<5 \times 10^{-8}$ and the $\mathrm{R}$ statistical software 151 package phenoscanner (https://github.com/phenoscanner/phenoscanner) was used to 152 download all data for our significant variants ${ }^{30}$. Chromatin state data based on 25-state 153 Imputation Based Chromatin State Model across 24 Blood, T-cell, HSC and B-cell lines 154 was downloaded from the Roadmap Epigenomics project 155 (https://egg2.wustl.edu/roadmap/data/byFileType/chromhmmSegmentations/ChmmMod 156 els/imputed12marks/jointModel/final/) ${ }^{31}$. Figures including chromatin state information 157 and results from previous GWAS were constructed using the R Bioconductor package 
158 gviz ${ }^{32-34}$. Lastly, we sought to identify promoter interaction regions (PIR), defined as

159 significant interactions between gene promotors and distal genomic regions. Variants in

160 PIRs can be connected to potential gene targets and thus can impact gene function ${ }^{34}$.

161 Briefly Hi-C libraries, enriched for promoter sequences, are generated with biotinylated

162 RNA baits complementary to the ends of promoter-containing restriction fragments.

163 Promoter fragments become bait for pieces of the genome that are targets with which

164 they frequently interact, allowing regulatory elements and enhancers to be pulled down

165 and sequenced. Statistical tests of bait-target pairs are done to define significant PIRs

166 and their targets $32,35,36$. To identify the genomic features with which our significant SNPs

167 might be interacting via chromatin looping we used publicly available Promoter Capture

$168 \mathrm{Hi}-\mathrm{C}(\mathrm{PCHi}-\mathrm{C})$ data on a lymphoblastoid cell line (LCL), GM12878, and two ex vivo CD34 ${ }^{+}$

169 hematopoietic progenitor cell lines (primary hematopoietic G-CSF mobilized stem cells

170 and hematopoietic stem cells) ${ }^{35}$. We integrated our SNP data with the PCHi-C cell line

171 data and visualized these interactions using circos plots ${ }^{37}$.

173 RESULTS

174 DISCOVeRY-BMT cases and controls

175 Results of quality control have been described elsewhere. ${ }^{14}$ Following quality control, the

176 DISCOVeRY-BMT cohorts include 1,769 AML and 540 MDS patients who received URD-

177 BMT as treatment and 2,814 unrelated donors as controls (Table 1). The majority of AML

178 cases are de novo $(\mathrm{N}=1618)$ with normal cytogenetics $(\mathrm{N}=543), 6 \%$ of patients had

179 therapy-related AML (t-AML). The most frequently reported previous cancers in patients 180 with t-AML were breast $(\mathrm{N}=51)$, non-Hodgkin Lymphoma (NHL), $\mathrm{N}=23, \mathrm{HL}(\mathrm{N}=14)$, 
181 Sarcoma $(\mathrm{N}=12)$, Gynecologic $(\mathrm{N}=8)$, Acute Lymphoblastic Leukemia $(\mathrm{N}=6)$ and

182 Testicular $(\mathrm{N}=6)$. Prior therapies for these patients were approximately equally divided

183 between single agent chemotherapy and combined modality chemotherapy plus

184 radiation. Almost half of MDS patients had Refractory Anemia with Excess Blasts (RAEB)

$185-1$ and RAEB-2. Of patients with t-MDS ( 18\% of MDS patients), $65 \%$ had antecedent

186 hematologic cancers or disorders. The most frequently reported antecedent cancers in

187 MDS patients were NHL ( $\mathrm{N}=27)$, breast $(\mathrm{N}=15)$, Acute Lymphoblastic Leukemia $(\mathrm{N}=8)$,

$188 \mathrm{HL}(\mathrm{N}=8)$, AML $(\mathrm{N}=8)$, Sarcoma $(\mathrm{N}=6)$ and $\mathrm{CLL}(\mathrm{N}=5)$ (Table 1). Overall, the distribution

189 of antecedent cancers differed significantly between t-MDS and t-AML, with almost 2/3 of

190 t-MDS and 1/3 of t-AML patients diagnosed with a prior hematologic cancer.

191

192 SNP Associations with AML and MDS

193 GWAS of AML by subtype (abnormal cytogenetics, normal cytogenetics and t-AML) and

194 MDS (de novo and t-MDS) are shown in Supplemental Figure 1. No population

195 stratification was observed in PCA analysis and $\lambda=1.0$ in both cohorts.

196 To identify loci that show association with AML and MDS we used ASSET. For SNPs to

197 be considered, we used previously defined criteria, which required ASSET SNP

198 associations at $P \leq 5.0 \times 10^{-8}$ with significant individual one-sided subset tests $(P<0.01)$,

199 the variant association could not be driven by a single disease nor could it be both 200 positively and negatively associated in different cohorts of the same disease. ${ }^{5}$ In the 201 ASSET GWAS analyses we identified a novel typed SNP associated with AML and MDS 202 on Chromosome 6 (Figure 1). The T allele at rs12203592, a variant in intron 4 of 203 Interferon Regulatory Factor 4 (IRF4), conferred increased risk of de novo abnormal 
204 cytogenetic AML, de novo normal cytogenetic AML, MDS and t-MDS (OR=1.38; 95\% Cl,

205 1.26-1.51, $P_{\text {meta }}=2.8 \times 10^{-12}$ ). T-AML showed no association with rs12203592. The effect

206 allele frequency was $19 \%$ in de novo AML, MDS and t-MDS cases versus $14 \%$ in controls.

207 ASSET analyses also identified another variant in modest linkage disequilibrium (LD),

$208 r^{2}=.7$, with $r s 12203592$ in the regulatory region of IRF4; the A allele at rs62389423,

209 showed a putative association with de novo AML and MDS (OR=1.36; 95\% Cl, 1.21-1.52,

$\left.210 \quad P_{\text {meta }}=1.2 \times 10^{-7}\right)($ Figure 2a).

211 We identified one significant association in the subtype GWAS which was disease

212 specific. The $\mathrm{C}$ allele in rs78898975 in TATA-box binding protein associated factor 2

213 (TAF2), associated with an increased risk of t-MDS $\left(\mathrm{OR}_{\text {meta }}=5.87,95 \% \mathrm{Cl}=3.20,10.76\right.$,

$\left.214 \mathrm{P}_{\text {meta }}=9.9 \times 10^{-9}\right)$ but not de novo MDS $\left(\mathrm{OR}=1.8,95 \% \mathrm{Cl}=.81,1.45, \mathrm{P}_{\text {meta }}=.20\right)$

215 (Supplemental Figure 1). The effect allele frequency was 7\% in t-MDS, 2\% in de novo

216 MDS and $1.5 \%$ in controls.

217 A previous genome-wide association study of AML done in European American cases

218 and controls reported a susceptibility variant in BICRA (rs75797233) ${ }^{38}$. The variant was

219 not significantly associated with $A M L$ risk in our meta-analyses $(\mathrm{OR}=1.08,95 \% \mathrm{Cl}=.78-$

220 1.37). However, their cohort did not include patients who received an allogeneic

221 transplant as curative therapy and the distribution of AML subtypes differed between the

222 studies. In addition, the lower frequency $(\mathrm{MAF}=.02)$ of this imputed this variant (info score

$223>.8$ in both cohorts) possibly reduced power to detect an effect. 
Multiple GWAS of healthy individuals have shown associations between the T allele at rs12203592 and higher eosinophil counts, lighter skin color, lighter hair, less tanning ability, and increased freckling. ${ }^{30,39}$ GWAS have also identified associations between this allele and increased risk of childhood acute lymphoblastic leukemia in males, nonmelanoma skin cancer, squamous cell carcinoma, cutaneous squamous cell carcinoma,

231 basal cell carcinoma, actinic keratosis, and progressive supranuclear palsy (Figure 2b). ${ }^{30}$

232 Furthermore, analyses of multiple B-cell malignancies recently identified a rs9392017,

233 adjacent to IRF4, as a pleiotropic susceptibility variant associated with both CLL and 234 Hodgkin Lymphoma(HL) $5,33,35,40$. This SNP is approximately $40 \mathrm{~Kb}$ away from 235 rs12203592, although not in LD $\left(r^{2}=.01\right)$.

236 The rs12203592 risk allele associated with increased expression of IRF4, $\mathrm{P}=1.48 \times 10^{-29}$ 237 in whole blood $^{28}$. IRF4 is a key transcription factor for lymphoid and myeloid 238 hematopoiesis ${ }^{41-44}$ and rs12203592 resides in a regulatory region across Blood, HSC, B-

239 Cell and T-Cell lines (Figure 2c). The variant's regulomedb score indicates how likely a 240 variant is to be a regulatory element from 1a (most likely) to 7 (no data); the variant's 241 score of $2 b$, indicates the variant is likely to affect transcription factor binding ${ }^{45}$. While the $242 \mathrm{HL}$ and CLL pleiotropic variant rs9392017 was not a significant eQTL for IRF4 in whole 243 blood, PCHi-C cell line data from both GM12878 and the ex vivo CD34+ hematopoietic 244 progenitor cell lines show chromatin looping between rs9392017 and the regulatory 245 region containing rs12203592 (Supplemental Figure 2).

246 The t-MDS associated C allele in rs78898975 is correlated with significantly lower 247 expression of TAF2 $\left(\mathrm{P}=1.95 \times 10^{-13}\right)$ and DEPTOR $\left(\mathrm{P}=4.7 \times 10^{-9}\right)$ gene expression in 248 whole blood. 28,46 


\section{Heritability estimates of AML and MDS}

251 The heritability of AML and MDS on the observed scale due to genotyped variants was significantly from a heritability of zero $\left(\mathrm{P}=2.0 \times 10^{-16}\right)$. The proportion of variance in de novo AML with normal cytogenetics and de novo MDS on the liability scale had similar

256 heritability at $9 \%, \mathrm{SE}=.03, \mathrm{P}=1.9 \times 10^{-3}$ and $14 \%, \mathrm{SE}=.04, \mathrm{P}=1.4 \times 10^{-4}$, respectively.

257 Treatment-related AML and MDS were tested independently and estimated proportion of variance explained by all SNPs was $7 \%$ for t-AML and $4 \%$ for $\mathrm{t}-\mathrm{MDS}$, however SE were high and the heritability did not significantly differ from zero.

\section{Transcriptome-wide association study - PrediXcan}

262 Using PrediXcan ${ }^{11}$ gene expression imputation models trained on the DGN data set, we

263 identified one transcriptome wide significant gene associated with de novo AML and MDS. Increased expression of IRF4 was associated with an increased risk for the

266 consistent with our SNP-level findings (Figure 3).

267 Whole blood transcriptome models also identified two additional genes with suggestive 268 associations with de novo AML and MDS. Increased expression of AKT Serine/Threonine

269 Kinase 1, AKT1 at 14q32.33 was associated with risk for the development of de novo 270 AML and MDS (OR=1.56; 95\% Cl, 1.25-1.95, $P_{\text {meta }}=1.0 \times 10^{-4}$ ) (Figure 4). Likewise,

271 increased expression of Ras guanyl nucleotide-releasing protein 2, RASGRP2, was 
272 associated with an increased risk for development of de novo AML and MDS (OR=4.05;

$\left.27395 \% \mathrm{Cl}, 1.84-8.91, \mathrm{P}_{\mathrm{meta}}=5 \times 10^{-4}\right)($ Figure 4).

DISCUSSION

276 We performed the first large scale AML and MDS GWAS in a URD-BMT population

277 providing evidence of novel pleotropic risk loci associated with increased susceptibility to

278 AML and MDS. We identified an association between the T allele at rs12203592 in IRF4

279 and an increased risk for the development of de novo AML, de novo MDS and t-MDS in

280 patients who had undergone URD-BMT compared to healthy donor controls. While

281 therapy-related myeloid neoplasms have been shown to be genetically and etiologically

282 similar to other high-risk myeloid neoplasms ${ }^{47}$, in our transplant population t-AML did not

283 associate with this variant, while t-MDS did show evidence of association with

284 rs12203592. We also identified a genome-wide significant t-MDS variant which was an

285 eQTL for both TAF2 and DEPTOR genes. We also provide the first estimates of the

286 heritability of AML and MDS, at between $9-14 \%$, which are in line with other GWAS of

287 cancer heritability on the liability scale, indicating that genetic variation contributes to AML

288 and MDS susceptibility. ${ }^{48}$

289 The rs12203592 SNP has been shown to regulate IRF4 transcription by physical

290 interaction with the IRF4 promoter through a chromatin loop ${ }^{49}$. This SNP resides in an

291 important position within NFkB motifs in multiple blood and immune cell lines, supporting

292 the hypothesis that this SNP may modulate NFkB repression of IRF4 expression. ${ }^{50,51}$

293 Furthermore, this SNP resides in a hematopoietic transcription factor that has been 
295 which we show interacts with the region containing our susceptibility variant. These data

296 add to the mounting evidence that there could be pleiotropic genes across multiple

297 hematologic cancers $5,52-55$.

298 Imputed gene expression logistic regression models showed a significant association

299 between higher predicted levels of IRF4 expression and the risk for development of de

300 novo AML or MDS ${ }^{11}$. Although IRF4 functions as a tumor suppressor gene in early B-cell

301 development 56 , in multiple myeloma IRF4 is a well-established oncogene ${ }^{44}$, with

302 oncogenic implications extending to adult leukemias ${ }^{57}$ and lymphomas ${ }^{58}$, as well as

303 pediatric leukemia. IRF4 overexpression is a hallmark of activated B-cell-like type of

304 diffuse large B-cell lymphoma and associated with classical Hodgkin lymphoma (cHL),

305 plasma cell myeloma and primary effusion lymphoma. ${ }^{59}$ In a case-control study of

306 childhood leukemia increased IRF4 expression was higher in immature B-common acute

307 lymphoblastic leukemia and T-cell leukemia with the highest expression levels in pediatric

308 AML patients compared to controls ${ }^{60}$. In addition to the CLL genetic susceptibility loci

309 identified in IRF4, high expression levels of the gene have been shown to correlate with

310 poor clinical prognosis ${ }^{61}$.

311 TWAS studies can be a powerful tool to help prioritize potentially causal genes. It is,

312 however, imperative to investigate the SNP and gene-expression associations in the

313 context of the surrounding variants and genes to reduce the possibility of a false signal

314 from co-regulation. Co-regulation can occur when there are multiple GWAS and TWAS

315 hits due to linkage disequilibrium and thus it becomes difficult to determine which locus

316 is driving the phenotypic association. In our study, the SNP rs12203592 is a significant

317 eQTL for only IRF4, this implies that the SNP and imputed gene expression signal we 
318 identified is not being driven by co-regulation of neighboring SNPs and/or genes. When

319 considering non-imputed gene expression sets, eQTLgen ${ }^{28}$ corroborates this finding;

320 rs12230592 is significantly associated with only increased expression of IRF4. In addition,

321 the relationship of rs12203592 to IRF4 expression in blood seems tissue specific, as

322 GTEx data across over 70 tissues shows association with only lung tissue at $P=9.1 \times 10^{-9}$.

323 The specificity of rs12203592 to IRF4 expression in blood and the lack of correlation

324 between IRF4 expression and other genes in DISCOVeRY-BMT give confidence that the

325 observed ASSET association is the potential susceptibility locus in the region. The

326 functional significance of variants in this gene in hematopoiesis and its previous

327 recognition as a locus associated with the risk for development of other hematological

328 malignancies, further strengthen the evidence of an association of IRF4 with development

329 of $A M L$ and MDS.

330 In addition to IRF4, we identified an association between the risk for development of de

331 novo AML or MDS and higher expression of $A K T 1$. AKT1 is an oncogene which plays a

332 critical role in the PI3KJAKT pathway. AML patients frequently show increased AKT1

333 activity, providing leukemic cells with growth and survival promoting signals ${ }^{62}$ and

334 enhanced $A K T$ activation has been implicated in the transformation from MDS to AML

335 and overexpression of $A K T$ has been shown to induce leukemia in mice. ${ }^{63}$

336 We also identified AML and MDS gene expression associations with RASGRP2, which is

337 expressed in various blood cell lineages and platelets, acts on the Ras-related protein

338 Rap and functions in platelet adhesion. GWAS have identified significant variants in this

339 gene associated with immature dendritic cells (\% CD32+) and immature fraction of

340 reticulocytes, a blood cell measurement shown to be elevated in patients with MDS 
341 versus controls. ${ }^{39}$ RASGRP2 expression has not been studied in relation to AML or MDS,

342 however recently RASGRP2/Rap1 signaling was shown to be functionally linked to the

343 CD38-associated increased CLL cell migration. The migration of CLL cells into lymphoid

344 tissues because of proliferation induced by B-cell receptor activation is thought to be an

345 important component of CLL pathogenesis. ${ }^{64}$ This finding has implications for the design

346 of novel treatments for CD38+ hematological diseases. ${ }^{64}$ These data imply the replication

347 of these gene expression associations with the development of AML and MDS are 348 warranted.

349 This is the largest genome-wide AML and MDS susceptibility study to date. Despite our 350 relatively large sample size, the complexity of cytogenetic risk groups in these diseases

351 limits our analysis, particularly with respect to therapy-related AML and MDS.

352 The DISCOVeRY-BMT study population is composed of mostly European American non-

353 Hispanics and thus validation of these associations in a non-white cohort of patients is

354 imperative. Lastly, the use of TWAS is a powerful way to start to prioritize causal genes

355 for follow-up after GWAS, however there are limitations. TWAS tests for association with

356 genetically predicted gene expression and not total gene expression, which includes

357 environmental, technical and genetic components. ${ }^{65}$

358 Our results provide evidence for the impact of common variants on the risk for AML or

359 MDS susceptibility and further characterization of the $6 \mathrm{p} 25.3$ locus might provide a more

360 mechanistic basis for the pleiotropic role of IRF4 in AML and MDS susceptibility. The co-

361 identification of variants in IRF4 associated with the risk for both myeloid and lymphoid

362 malignancy supports the importance of broader studies that span the spectrum

363 hematologic malignancies. 


\section{ACKNOWLEDGEMENTS / CONTRIBUTIONS / FUNDING}

This work was supported by grants from the National Institute of Health. LESC and TH were supported by $1 \mathrm{R} 01 \mathrm{HL} 102278$ and $1 \mathrm{R} 03 \mathrm{CA} 188733$ to perform this work. EK is supported by the Pelotonia Foundation Graduate Student Fellowship. Any opinions, findings, and conclusions expressed in this material are those of the author(s) and do not necessarily reflect those of the Pelotonia Fellowship Program or The Ohio State University.

ACG was supported by CA9204, Mayo Clinic R25 Training Grant when she performed a majority of this work. The CIBMTR is supported by Public Health Service Grant/Cooperative Agreement 5U24-CA076518 from the National Cancer Institute ( $\mathrm{NCl}$ ), the National Heart, Lung and Blood Institute (NHLBI) and the National Institute of Allergy and Infectious Diseases (NIAID); a Grant/Cooperative Agreement 5U10HL069294 from NHLBI and NCl; a contract HHSH250201200016C with Health Resources and Services Administration (HRSA/DHHS); two Grants N00014-15-1-0848 and N00014-16-1-2020 from the Office of Naval Research; and grants from Alexion; *Amgen, Inc.; Anonymous donation to the Medical College of Wisconsin; Astellas Pharma US; AstraZeneca; Be the Match Foundation; $\quad$ *Bluebird Bio, Inc.; *Bristol Myers Squibb Oncology; *Celgene Corporation; Cellular Dynamics International, Inc.; *Chimerix, Inc.; Fred Hutchinson Cancer Research Center; Gamida Cell Ltd.; Genentech, Inc.; Genzyme Corporation; *Gilead Sciences, Inc.; Health Research, Inc. Roswell Park Cancer Institute; HistoGenetics, Inc.; Incyte Corporation; Janssen Scientific Affairs, LLC; *Jazz Pharmaceuticals, Inc.; Jeff Gordon Children's Foundation; The Leukemia \& Lymphoma 
Society; Medac, GmbH; Medlmmune; The Medical College of Wisconsin; *Merck \& Co, Inc.; Mesoblast; MesoScale Diagnostics, Inc.; *Miltenyi Biotec, Inc.; National Marrow Donor Program; Neovii Biotech NA, Inc.; Novartis Pharmaceuticals Corporation; Onyx Pharmaceuticals; Optum Healthcare Solutions, Inc.; Otsuka America Pharmaceutical, Inc.; Otsuka Pharmaceutical Co, Ltd. - Japan; PCORI; Perkin Elmer, Inc.; Pfizer, Inc; *Sanofi US; *Seattle Genetics; *Spectrum Pharmaceuticals, Inc.; St. Baldrick's Foundation; *Sunesis Pharmaceuticals, Inc.; Swedish Orphan Biovitrum, Inc.; Takeda Oncology; Telomere Diagnostics, Inc.; University of Minnesota; and *Wellpoint, Inc. The views expressed in this article do not reflect the official policy or position of the National Institute of Health, the Department of the Navy, the Department of Defense, Health Resources and Services Administration (HRSA) or any other agency of the U.S. Government.

${ }^{*}$ Corporate Members

\section{Authorship Contributions}

J.W, A.C-G, L.S-C, and T.E.H designed the research, performed research and analysis, and wrote the manuscript.

C.A.H, D.V, X.S and L.P performed the genotyping.

X.Z., L.P, A.W and G.B performed quality control of genomic data.

All authors reviewed and approved the manuscript. 


\section{REFERENCES}

1. Walker CJ, Oakes CC, Genutis LK, et al. Genome-wide association study identifies an acute myeloid leukemia susceptibility locus near BICRA. Leukemia. 2019;33(3):771-775.

2. Knight JA, Skol AD, Shinde A, et al. Genome-wide association study to identify novel loci associated with therapy-related myeloid leukemia susceptibility. Blood. 2009;113(22):5575-5582.

3. Lv H, Zhang M, Shang Z, et al. Genome-wide haplotype association study identify the FGFR2 gene as a risk gene for acute myeloid leukemia. Oncotarget. 2017;8(5):7891-7899.

4. Bhattacharjee S, Rajaraman P, Jacobs KB, et al. A subset-based approach improves power and interpretation for the combined analysis of genetic association studies of heterogeneous traits. Am J Hum Genet. 2012;90(5):821-835.

5. Law PJ, Sud A, Mitchell JS, et al. Genome-wide association analysis of chronic lymphocytic leukaemia, Hodgkin lymphoma and multiple myeloma identifies pleiotropic risk loci. Sci Rep. 2017;7:41071.

6. Went $\mathrm{M}$, Sud A, Speedy $\mathrm{H}$, et al. Genetic correlation between multiple myeloma and chronic lymphocytic leukaemia provides evidence for shared aetiology. Blood Cancer J. 2018;9(1):1.

7. Churpek JE. Familial myelodysplastic syndrome/acute myeloid leukemia. Best Pract Res Clin Haematol. 2017;30(4):287-289.

8. Gao J, Gentzler RD, Timms AE, et al. Heritable GATA2 mutations associated with familial AMLMDS: a case report and review of literature. J Hematol Oncol. 2014;7:36.

9. Goldin LR, Kristinsson SY, Liang XS, Derolf AR, Landgren O, Bjorkholm M. Familial aggregation of acute myeloid leukemia and myelodysplastic syndromes. J Clin Oncol. 2012;30(2):179-183.

10. Gusev A, Ko A, Shi H, et al. Integrative approaches for large-scale transcriptome-wide association studies. Nat Genet. 2016;48(3):245-252.

11. Gamazon ER, Wheeler HE, Shah KP, et al. A gene-based association method for mapping traits using reference transcriptome data. Nat Genet. 2015;47(9):1091-1098.

12. Hahn T, Sucheston-Campbell LE, Preus L, et al. Establishment of Definitions and Review Process for Consistent Adjudication of Cause-specific Mortality after Allogeneic Unrelated-donor Hematopoietic Cell Transplantation. Biol Blood Marrow Transplant. 2015;21(9):1679-1686.

13. Clay-Gilmour Al, Hahn T, Preus LM, et al. Genetic association with B-cell acute lymphoblastic leukemia in allogeneic transplant patients differs by age and sex. Blood Adv. 2017;1(20):1717-1728.

14. Karaesmen E, Rizvi AA, Preus LM, et al. Replication and validation of genetic polymorphisms associated with survival after allogeneic blood or marrow transplant. Blood. 2017;130(13):1585-1596.

15. Zhu Q, Yan L, Liu Q, et al. Exome chip analyses identify genes affecting mortality after HLAmatched unrelated-donor blood and marrow transplantation. Blood. 2018;131(22):2490-2499.

16. Yan L, Ma C, Wang D, et al. OSAT: a tool for sample-to-batch allocations in genomics experiments. BMC Genomics. 2012;13:689.

17. Price AL, Patterson NJ, Plenge RM, Weinblatt ME, Shadick NA, Reich D. Principal components analysis corrects for stratification in genome-wide association studies. Nat Genet. 2006;38(8):904-909. 18. Das S, Forer L, Schonherr S, et al. Next-generation genotype imputation service and methods. Nat Genet. 2016;48(10):1284-1287.

19. McCarthy S, Das S, Kretzschmar W, et al. A reference panel of 64,976 haplotypes for genotype imputation. Nat Genet. 2016;48(10):1279-1283.

20. Lee $\mathrm{CH}$, Eskin $\mathrm{E}$, Han B. Increasing the power of meta-analysis of genome-wide association studies to detect heterogeneous effects. Bioinformatics. 2017;33(14):i379-i388. 
21. Deary IJ, Yang J, Davies G, et al. Genetic contributions to stability and change in intelligence from childhood to old age. Nature. 2012;482(7384):212-215.

22. Lee SH, Yang J, Goddard ME, Visscher PM, Wray NR. Estimation of pleiotropy between complex diseases using single-nucleotide polymorphism-derived genomic relationships and restricted maximum likelihood. Bioinformatics. 2012;28(19):2540-2542.

23. Yang J, Lee SH, Goddard ME, Visscher PM. GCTA: a tool for genome-wide complex trait analysis. Am J Hum Genet. 2011;88(1):76-82.

24. Mitchell JS, Johnson DC, Litchfield K, et al. Implementation of genome-wide complex trait analysis to quantify the heritability in multiple myeloma. Sci Rep. 2015;5:12473.

25. Lu Y, Ek WE, Whiteman D, et al. Most common 'sporadic' cancers have a significant germline genetic component. Hum Mol Genet. 2014;23(22):6112-6118.

26. Lee $\mathrm{SH}$, Harold D, Nyholt DR, et al. Estimation and partitioning of polygenic variation captured by common SNPs for Alzheimer's disease, multiple sclerosis and endometriosis. Hum Mol Genet. 2013;22(4):832-841.

27. Battle A, Mostafavi S, Zhu X, et al. Characterizing the genetic basis of transcriptome diversity through RNA-sequencing of 922 individuals. Genome Res. 2014;24(1):14-24.

28. Võsa U, Claringbould A, Westra H-J, et al. Unraveling the polygenic architecture of complex traits using blood eQTL metaanalysis. bioRxiv. 2018:447367.

29. Carithers LJ, Ardlie K, Barcus M, et al. A Novel Approach to High-Quality Postmortem Tissue Procurement: The GTEx Project. Biopreserv Biobank. 2015;13(5):311-319.

30. Staley JR, Blackshaw J, Kamat MA, et al. PhenoScanner: a database of human genotypephenotype associations. Bioinformatics. 2016;32(20):3207-3209.

31. Roadmap Epigenomics C, Kundaje A, Meuleman W, et al. Integrative analysis of 111 reference human epigenomes. Nature. 2015;518(7539):317-330.

32. Cairns J, Freire-Pritchett $P$, Wingett SW, et al. CHiCAGO: robust detection of DNA looping interactions in Capture Hi-C data. Genome Biol. 2016;17(1):127.

33. Mifsud B, Tavares-Cadete F, Young AN, et al. Mapping long-range promoter contacts in human cells with high-resolution capture Hi-C. Nat Genet. 2015;47(6):598-606.

34. Spurrell CH, Dickel DE, Visel A. The Ties That Bind: Mapping the Dynamic Enhancer-Promoter Interactome. Cell. 2016;167(5):1163-1166.

35. Schofield EC, Carver T, Achuthan P, et al. CHiCP: a web-based tool for the integrative and interactive visualization of promoter capture Hi-C datasets. Bioinformatics. 2016;32(16):2511-2513.

36. Schoenfelder S, Javierre BM, Furlan-Magaril M, Wingett SW, Fraser P. Promoter Capture Hi-C: High-resolution, Genome-wide Profiling of Promoter Interactions. J Vis Exp. 2018(136).

37. Yu Y, Ouyang Y, Yao W. shinyCircos: an R/Shiny application for interactive creation of Circos plot. Bioinformatics. 2018;34(7):1229-1231.

38. Walker CJ, Oakes CC, Genutis LK, et al. Genome-wide association study identifies an acute myeloid leukemia susceptibility locus near BICRA. Leukemia. 2018.

39. Astle WJ, Elding H, Jiang T, et al. The Allelic Landscape of Human Blood Cell Trait Variation and Links to Common Complex Disease. Cell. 2016;167(5):1415-1429 e1419.

40. Di Bernardo MC, Crowther-Swanepoel D, Broderick P, et al. A genome-wide association study identifies six susceptibility loci for chronic lymphocytic leukemia. Nat Genet. 2008;40(10):1204-1210.

41. Havelange V, Pekarsky Y, Nakamura T, et al. IRF4 mutations in chronic lymphocytic leukemia. Blood. 2011;118(10):2827-2829.

42. Pratt G, Fenton JA, Allsup D, et al. A polymorphism in the 3' UTR of IRF4 linked to susceptibility and pathogenesis in chronic lymphocytic leukaemia and Hodgkin lymphoma has limited impact in multiple myeloma. Br J Haematol. 2010;150(3):371-373. 
43. Salaverria I, Philipp C, Oschlies I, et al. Translocations activating IRF4 identify a subtype of germinal center-derived B-cell lymphoma affecting predominantly children and young adults. Blood. 2011;118(1):139-147.

44. Shaffer AL, Emre NC, Lamy L, et al. IRF4 addiction in multiple myeloma. Nature. 2008;454(7201):226-231.

45. Boyle AP, Hong EL, Hariharan M, et al. Annotation of functional variation in personal genomes using RegulomeDB. Genome Res. 2012;22(9):1790-1797.

46. Kamat MA, Blackshaw JA, Young R, et al. PhenoScanner V2: an expanded tool for searching human genotype-phenotype associations. Bioinformatics. 2019.

47. McNerney ME, Godley LA, Le Beau MM. Therapy-related myeloid neoplasms: when genetics and environment collide. Nat Rev Cancer. 2017;17(9):513-527.

48. Sampson JN, Wheeler WA, Yeager M, et al. Analysis of Heritability and Shared Heritability Based on Genome-Wide Association Studies for Thirteen Cancer Types. J Natl Cancer Inst. 2015;107(12):djv279.

49. Visser M, Palstra RJ, Kayser M. Allele-specific transcriptional regulation of IRF4 in melanocytes is mediated by chromatin looping of the intronic rs12203592 enhancer to the IRF4 promoter. Hum Mol Genet. 2015;24(9):2649-2661.

50. Kheradpour P, Kellis M. Systematic discovery and characterization of regulatory motifs in ENCODE TF binding experiments. Nucleic Acids Res. 2014;42(5):2976-2987.

51. Ward LD, Kellis M. HaploReg: a resource for exploring chromatin states, conservation, and regulatory motif alterations within sets of genetically linked variants. Nucleic Acids Res. 2012;40(Database issue):D930-934.

52. Mitchell JS, Li N, Weinhold N, et al. Genome-wide association study identifies multiple susceptibility loci for multiple myeloma. Nat Commun. 2016;7:12050.

53. Vijayakrishnan J, Qian M, Studd JB, et al. Identification of four novel associations for B-cell acute lymphoblastic leukaemia risk. Nat Commun. 2019;10(1):5348.

54. Went M, Sud A, Speedy H, et al. Genetic correlation between multiple myeloma and chronic lymphocytic leukaemia provides evidence for shared aetiology. Blood Cancer J. 2018;9(1):1.

55. Slager SL, Camp NJ, Conde L, et al. Common variants within 6p21.31 locus are associated with chronic lymphocytic leukaemia and, potentially, other non-Hodgkin lymphoma subtypes. Br J Haematol. 2012;159(5):572-576.

56. Acquaviva J, Chen X, Ren R. IRF-4 functions as a tumor suppressor in early B-cell development. Blood. 2008;112(9):3798-3806.

57. De Silva NS, Simonetti G, Heise N, Klein U. The diverse roles of IRF4 in late germinal center B-cell differentiation. Immunol Rev. 2012;247(1):73-92.

58. Bisig B, Gaulard P, de Leval L. New biomarkers in T-cell lymphomas. Best Pract Res Clin Haematol. 2012;25(1):13-28.

59. Carbone A, Gloghini A, Aldinucci D, Gattei V, Dalla-Favera R, Gaidano G. Expression pattern of MUM1/IRF4 in the spectrum of pathology of Hodgkin's disease. Br J Haematol. 2002;117(2):366-372.

60. Adamaki M, Lambrou GI, Athanasiadou A, Tzanoudaki M, Vlahopoulos S, Moschovi M. Implication of IRF4 aberrant gene expression in the acute leukemias of childhood. PLoS One. 2013;8(8):e72326.

61. Allan JM, Sunter NJ, Bailey JR, et al. Variant IRF4/MUM1 associates with CD38 status and treatment-free survival in chronic lymphocytic leukaemia. Leukemia. 2010;24(4):877-881.

62. Tang Y, Halvarsson C, Nordigarden A, et al. Coexpression of hyperactivated AKT1 with additional genes activated in leukemia drives hematopoietic progenitor cells to cell cycle block and apoptosis. Exp Hematol. 2015;43(7):554-564. 
63. Kharas MG, Okabe R, Ganis JJ, et al. Constitutively active AKT depletes hematopoietic stem cells and induces leukemia in mice. Blood. 2010;115(7):1406-1415.

64. Mele S, Devereux S, Pepper AG, Infante E, Ridley AJ. Calcium-RasGRP2-Rap1 signaling mediates CD38-induced migration of chronic lymphocytic leukemia cells. Blood Adv. 2018;2(13):1551-1561.

65. Wainberg M, Sinnott-Armstrong N, Mancuso N, et al. Opportunities and challenges for transcriptome-wide association studies. Nature Genetics. 2019;51(4):592-599. 


\section{Figure Legends}

Figure 1. ASSET analysis and associations by AML and MDS subgroup

Forest plot of the odds ratios (OR) for the association between rs12203592 in IRF4 and MDS and AML subtypes. The variant resides in the Chromosome 6 outside the major histocompatibility complex region. Studies were weighted by inverse of the variance of the log (OR). The solid grey vertical line is positioned at the null value $(O R=1)$; values to the right represent risk increasing odds ratios. Horizontal lines show the $95 \% \mathrm{Cl}$ and the box is the OR point estimate for each casecontrol subset with its area proportional to the weight of the patient group. The diamond is the overall effect estimated by ASSET, with the $95 \% \mathrm{Cl}$ given by its width.

\section{Figure 2. IRF4 region with AML and MDS associated SNP p-values annotated with previous GWAS and Roadmap Epigenome Chromatin States.}

A. ASSET analysis AML and MDS SNP associations in the IRF4 region. The x-axis is the chromosome position in kilobase pairs and y-axis shows the -log10 ( $p$-values) for de novo AML and MDS susceptibility. The associated SNPs in the IRF4 region, rs12203592 and rs62389423, are highlighted with sky blue lines drawn through the point to show the relationship of the variant to GWAS hits and Roadmap Epigenome data (2C). rs12203592 and rs62389423 show moderate linkage disequilibrium $\left(r^{2}=0.7\right)$; rs62389423 and rs62389424 are almost perfectly correlated $\left(r^{2}=.95\right)$.

B. Previously reported GWAS SNPs in the IRF4 region. Phenotypes are color coded and all variants are associated at $\mathrm{P}<5 \times 10^{-8}$.

C. Genes in the region annotated with the chromatin-state segmentation track (ChromHMM) from Roadmap Epigenome data for all blood, T-cell, HSC and B-cells. The cell line numbers shown down the left side correspond to specific epigenome road map cell lines. E029:Primary monocytes from peripheral blood; E030:Primary neutrophils from peripheral blood; E031:Primary B cells from cord blood; E032:Primary B Cells from peripheral blood; E033:Primary T Cells from cord blood; E034:Primary T Cells from blood; E035:Primary hematopoietic stem cells; E036:Primary hematopoietic stem cells short term culture; E037:Primary T helper memory cells from peripheral blood 2; E038:Primary T help naïve cells from peripheral blood; E039:Primary T helper naïve cells from peripheral blood; E040:Primary Thelper memory cells from peripheral blood 1; E041:Primary $\mathrm{T}$ helper cells PMA-lonomycin stimulated; E042:Primary $\mathrm{T}$ helper 17 cells PMA-lonomycin stimulated; E043:Primary T helper cells from peripheral blood; E044:Primary T regulatory cells from peripheral blood; E045:Primary T cells effector/memory enriched from peripheral blood; E046:Primary Natural Killer cells from peripheral blood; E047:Primary T CD8 naïve cells from peripheral blood; E048:Primary T CD8 memory cells from peripheral blood; E-50:Primary hematopoietic stem cells G-CSF mobilized Female; E-51:Primary hematopoietic stem cells GCSF mobilized Male; E062:Primary Mononuclear Cells from Peripheral Blood; E0116 Lymphoblastic Cell Line. The colors indicate chromatin states imputed by ChromHMM and shown in the key titled "Roadmap Chromatin State"

\section{Figure 3. Manhattan plot of the de novo AML and MDS GWAS and TWAS.}

The plot represents the TWAS P-values (top) of each gene and de novo AML and MDS GWAS P-values (bottom) of each SNP included in the case-control association study. Significant and suggestive genes are highlighted in orange and labelled by their gene symbols. The orange horizontal line on the top represents the transcriptome-wide significance threshold of $P=4.5 \times 10^{-6}$. The orange horizontal line on the bottom represents the genome-wide threshold of $P=5.0 \times 10^{-8}$. 
Figure 4. Regional plots of PrediXcan-TWAS and SNP associations with AML and MDS Each box represents PrediXcan-TWAS significant genes AKT1, IRF4 and RASGRP2 +/- 0.5 megabases. The grey shaded bars represent the gene, where height is gene expression association and width is gene region in base pairs and the purple dots represent SNP associations with AML and MDS -log10 (P-values) are shown on the y-axis. Green and red lines denote the transcriptome-wide and genome wide significant $\mathrm{P}$-values, respectively. 


\begin{tabular}{|c|c|c|}
\hline Patient and Donor Characteristics & $\begin{array}{c}\text { Cases } \\
\text { Cohort } 1 / \text { Cohort } 2 \\
\mathrm{~N}=1627(\%) / 682(\%)\end{array}$ & $\begin{array}{c}\text { Controls } \\
\text { Cohort } 1 / \text { Cohort } 2 \\
\mathrm{~N}=2052(\%) / 762(\%)\end{array}$ \\
\hline \multicolumn{3}{|l|}{ Age, years } \\
\hline Median (range) & $50(<1-74.5) / 52(<1-78)$ & $33(18-61) / 31(18-60)$ \\
\hline \multicolumn{3}{|l|}{ Sex } \\
\hline Females & $741(46) / 312(46)$ & $656(32) / 209(27)$ \\
\hline \multicolumn{3}{|l|}{ Disease } \\
\hline$A M L$, all cases & $1282(79) / 487$ (71) & - \\
\hline de novo $A M L$ & $1164(72) / 454(66)$ & - \\
\hline de novo AML with normal cytogenetics & $373(23) / 170(25)$ & - \\
\hline de novo AML with abnormal cytogenetics & $595(37) / 241(35)$ & - \\
\hline $\begin{array}{l}\text { By Cytogenetic Subtype: } \\
\text { Core Binding Factor } \\
\text { MLL } \\
\text { Ph } \mathrm{t}(9 ; 22) \\
\text { APL } \mathrm{t}(15 ; 17) \\
\text { Any translocation } \\
\text { Trisomy } 8 \\
\text { Trisomy } 13,21 \text { or } 22 \\
\text { del5/del7 } \\
\text { Any Trisomy } \\
\text { Any Monosomy } \\
>3 \text { cytogenetic abnormalities }\end{array}$ & $\begin{array}{c}67(11) / 32(13) \\
72(12) / 48(20) \\
5(1) / 1(0) \\
18(3) / 3(1) \\
97(15) / 35(15) \\
103(17) / 22(9) \\
52(9) / 24(9) \\
123(21) / 55(23) \\
195(33) / 92(38) \\
153(26) / 50(21) \\
213(36) / 88(37)\end{array}$ & $\begin{array}{l}- \\
- \\
- \\
- \\
- \\
- \\
- \\
- \\
- \\
-\end{array}$ \\
\hline therapy-related $A M L$ & $113(7) / 33(5)$ & - \\
\hline $\begin{array}{l}\text { By Prior Diagnosis }{ }^{2} \text { : } \\
\text { Breast Cancer } \\
\text { Non-Hodgkin Lymphoma } \\
\text { Hodgkin Lymphoma } \\
\text { Sarcoma } \\
\text { Gynecologic Cancer } \\
\text { Acute Lymphoblastic Leukemia } \\
\text { Testicular Cancer } \\
\text { Other Disease }\end{array}$ & $\begin{array}{l}39(35) / 12(36) \\
20(18) / 3(9) \\
11(10) / 3(9) \\
9(3) / 8(9) \\
6(5) / 2(6) \\
4(4) / 2(6) \\
4(4) / 2(6) \\
20(18) / 4(12)\end{array}$ & $\begin{array}{l}- \\
- \\
- \\
- \\
- \\
- \\
-\end{array}$ \\
\hline
\end{tabular}




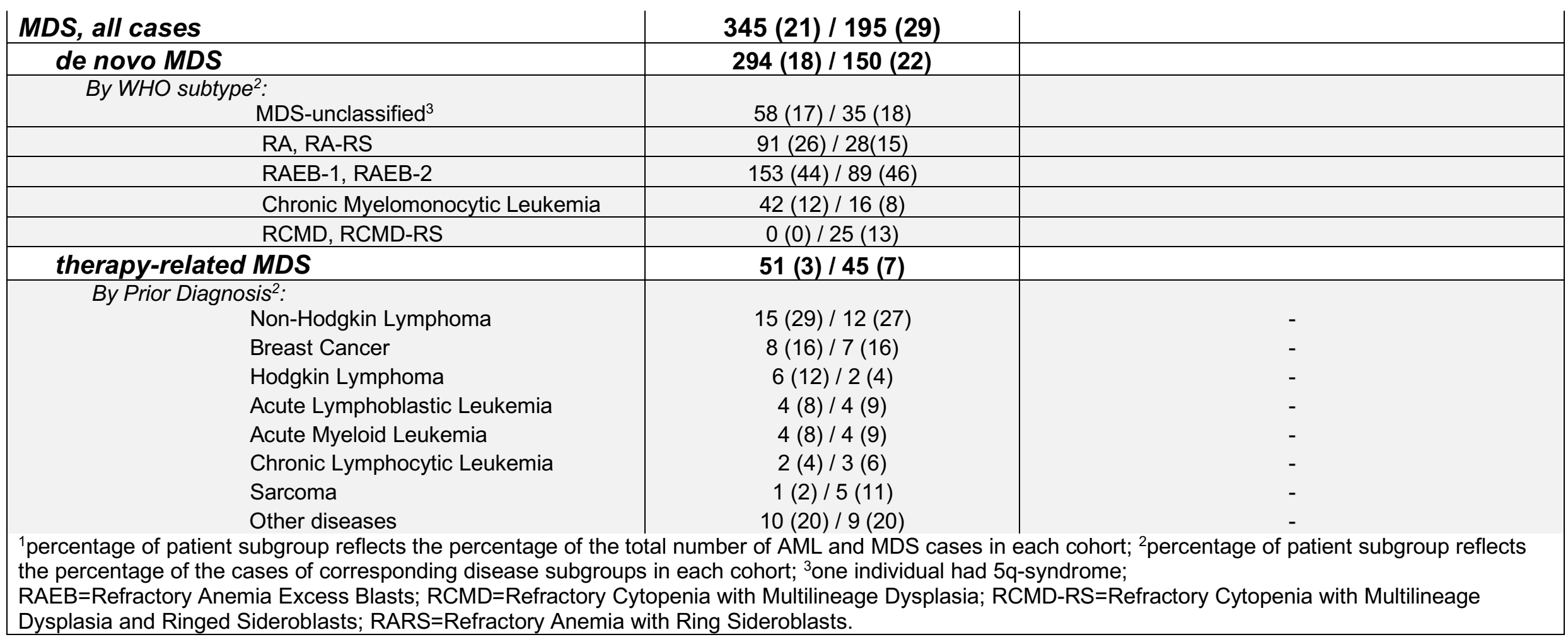


Figure 1. ASSET analysis and associations by AML and MDS subgroup

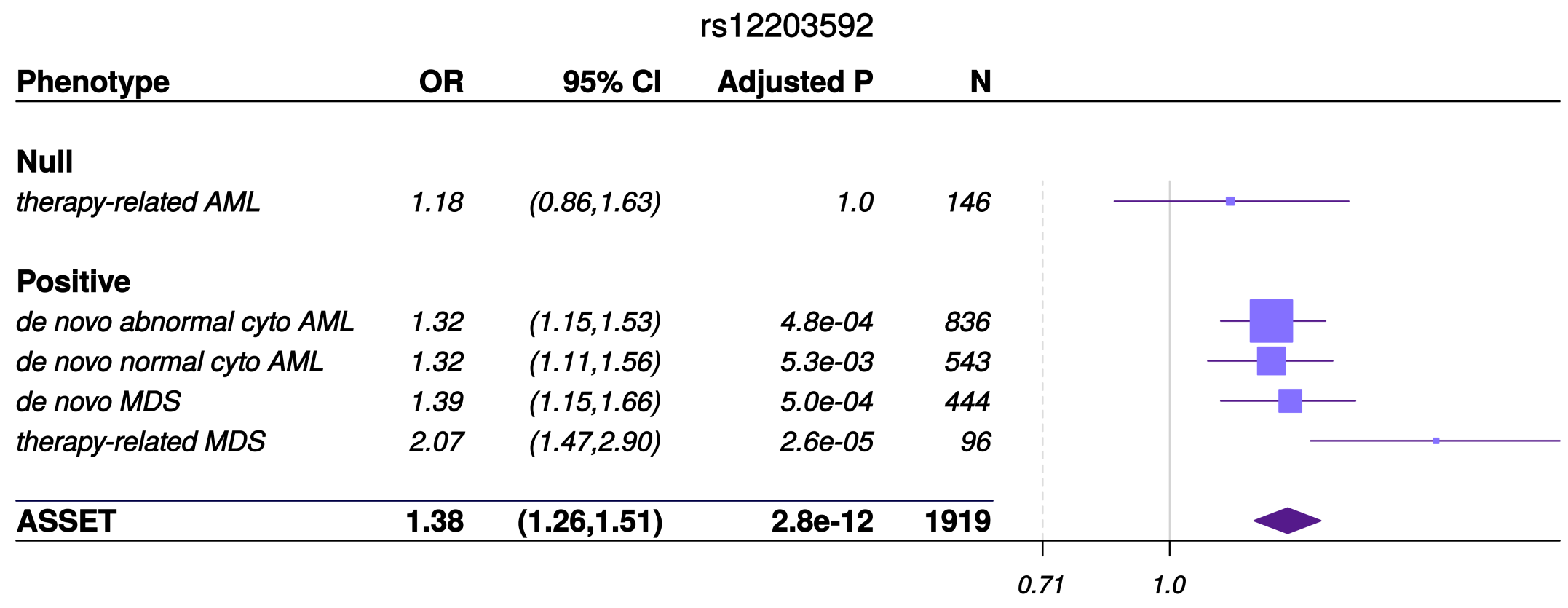


Figure 2. IRF4 region with AML and MDS associated SNP p-values annotated with previous GWAS and Roadmap Epigenome Chromatin States.

Chromosome 6

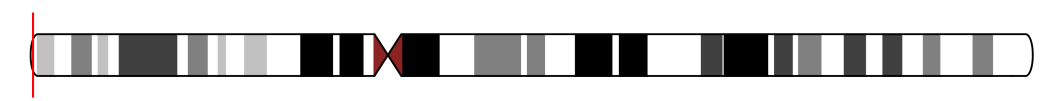

A

B
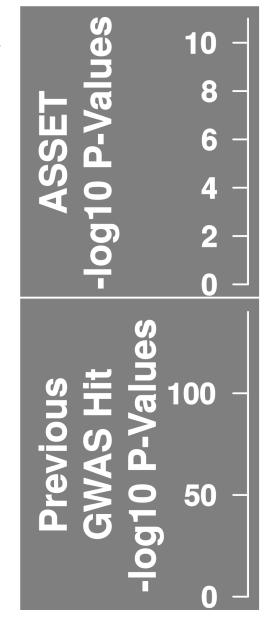
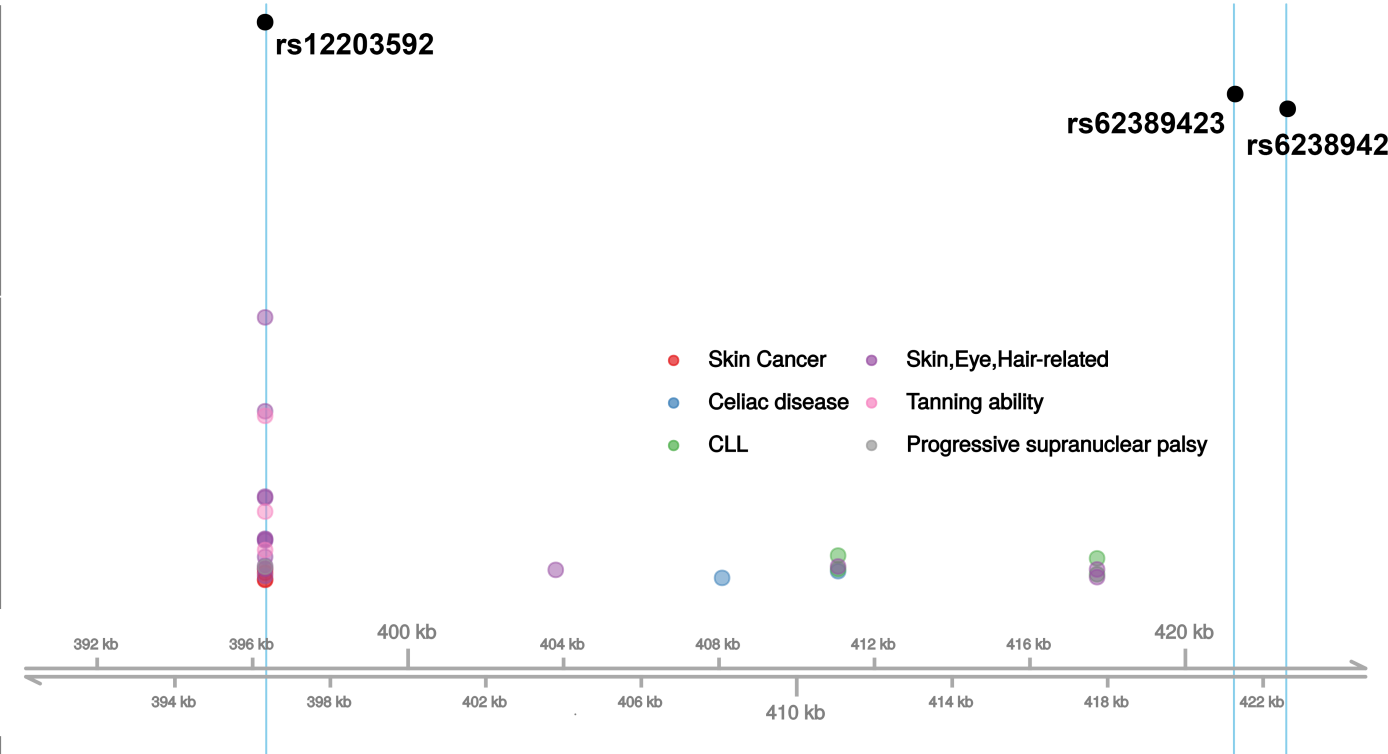

rs12203592

- Skin Cancer - Skin,Eye,Hair-related

- Celiac disease - Tanning ability

- CLL P Progressive supranuclear palsy

\section{홍 웅}

C

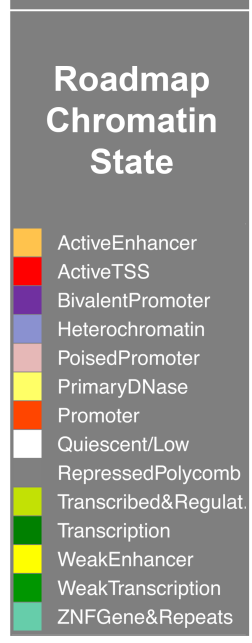

IRF4

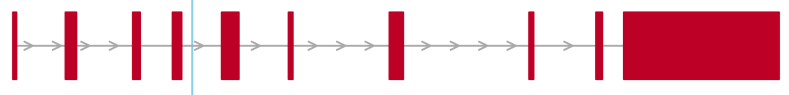

E029
E030

E031

E032

E033

E034
E035

E036
E037

E038
E039

E040

E041

E042

E043
E044

E044
E045

E046

E047

$E 047$
E048

E050
E051

E051

E116

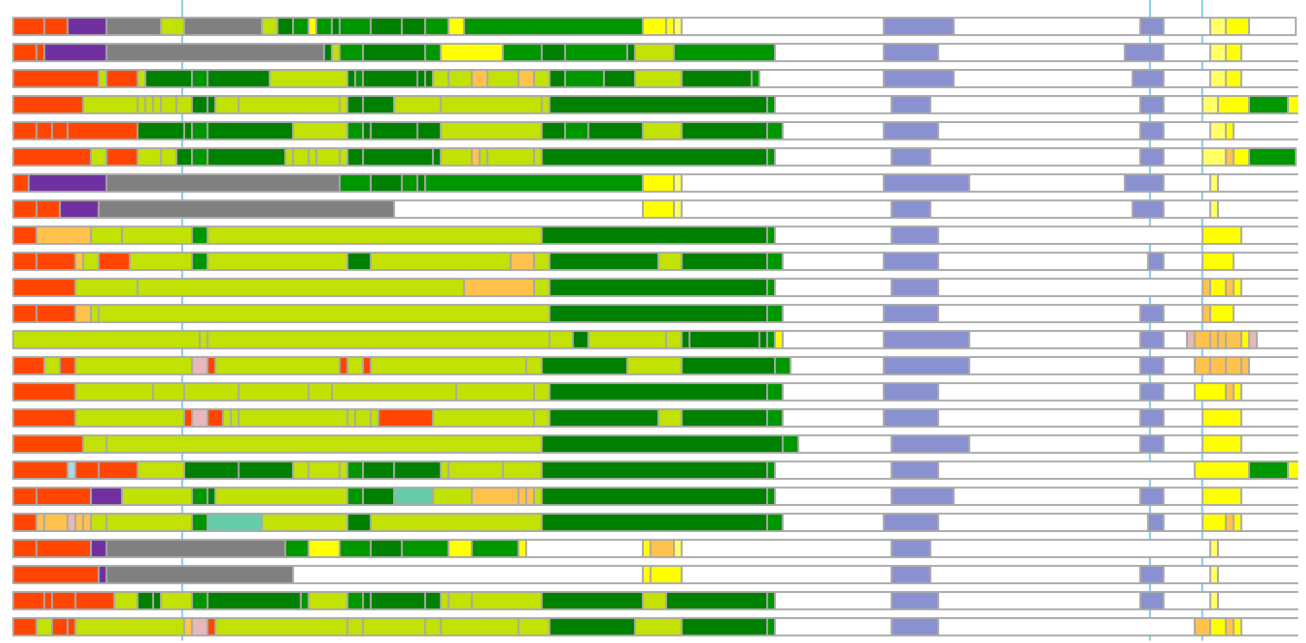


Figure 3. Manhattan plot of the de novo AML and MDS GWAS and TWAS.

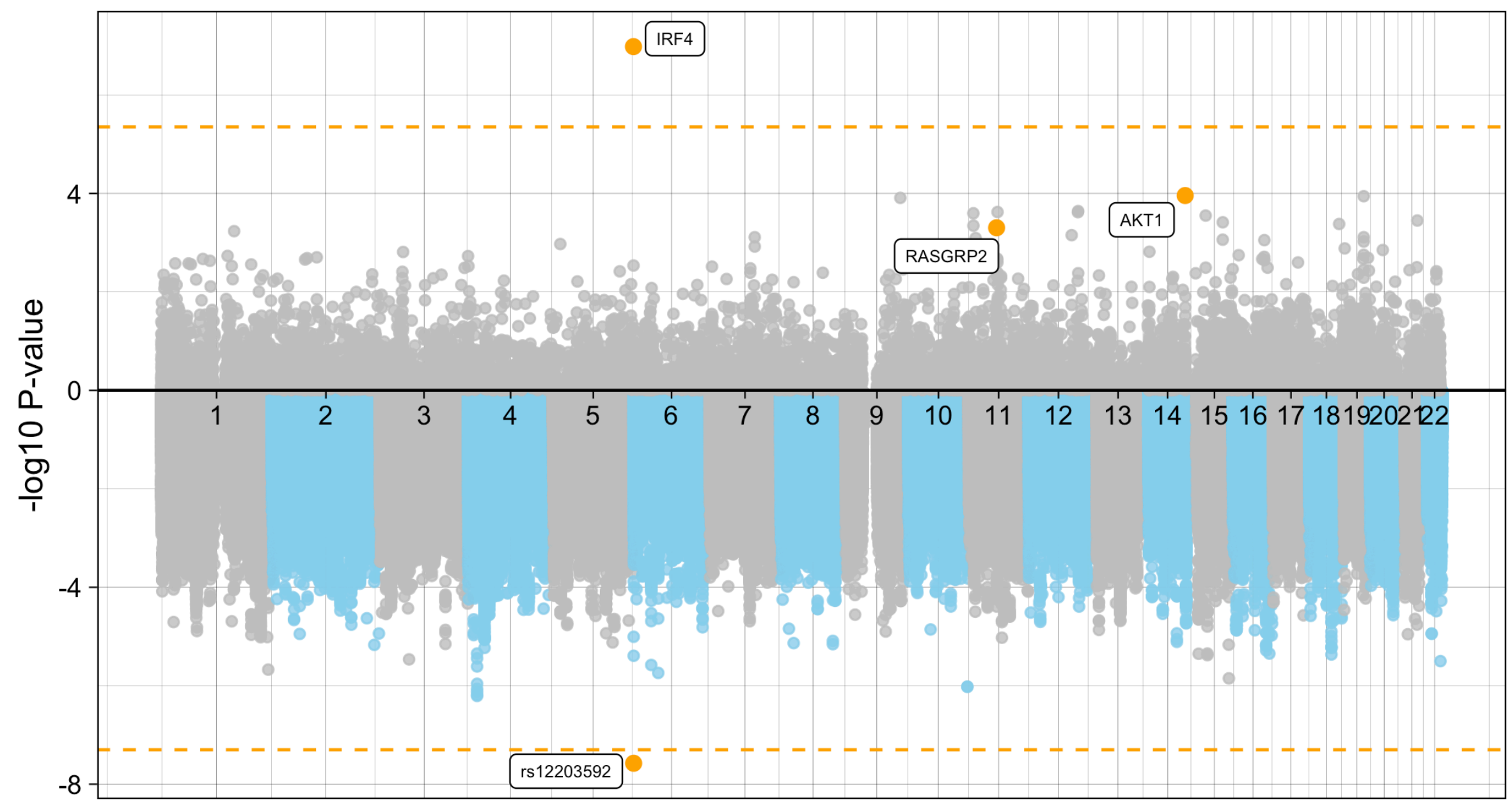

Chromosome Position 
Figure 4. Regional plots of PrediXcan-TWAS and SNP associations with AML and MDS

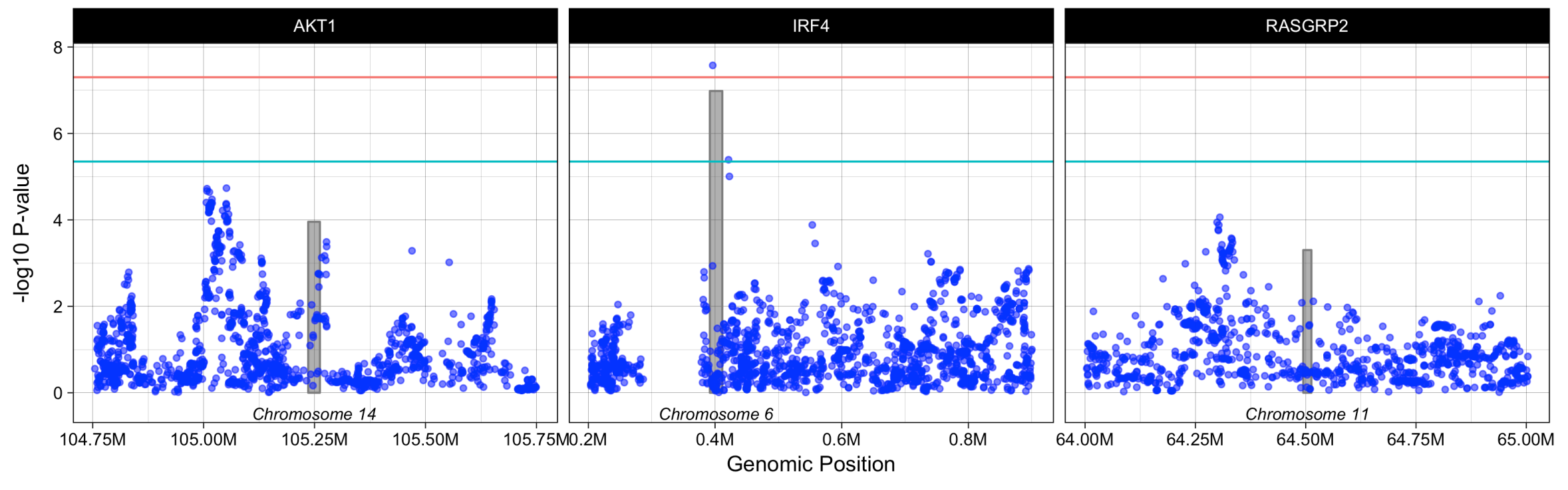

_ GW Significant — TW Significant 


\section{Supplemental Figure 1. Genome wide associations by cytogenetic subtype in DISCOVeRY-BMT}

Shown are the genome-wide $P$ values by subtype from the meta-analysis of DISCOVeRY-BMT cohorts, including a total of $2158 \mathrm{AML}$ and MDS cases and 2814 controls. The dashed horizontal line represents the suggestive threshold of $P=5.0 \times 10^{-6}$. The orange horizontal line represents the genome-wide significance threshold of $P=5.0 \times 10^{-8}$.

GWAS in individual subgroups
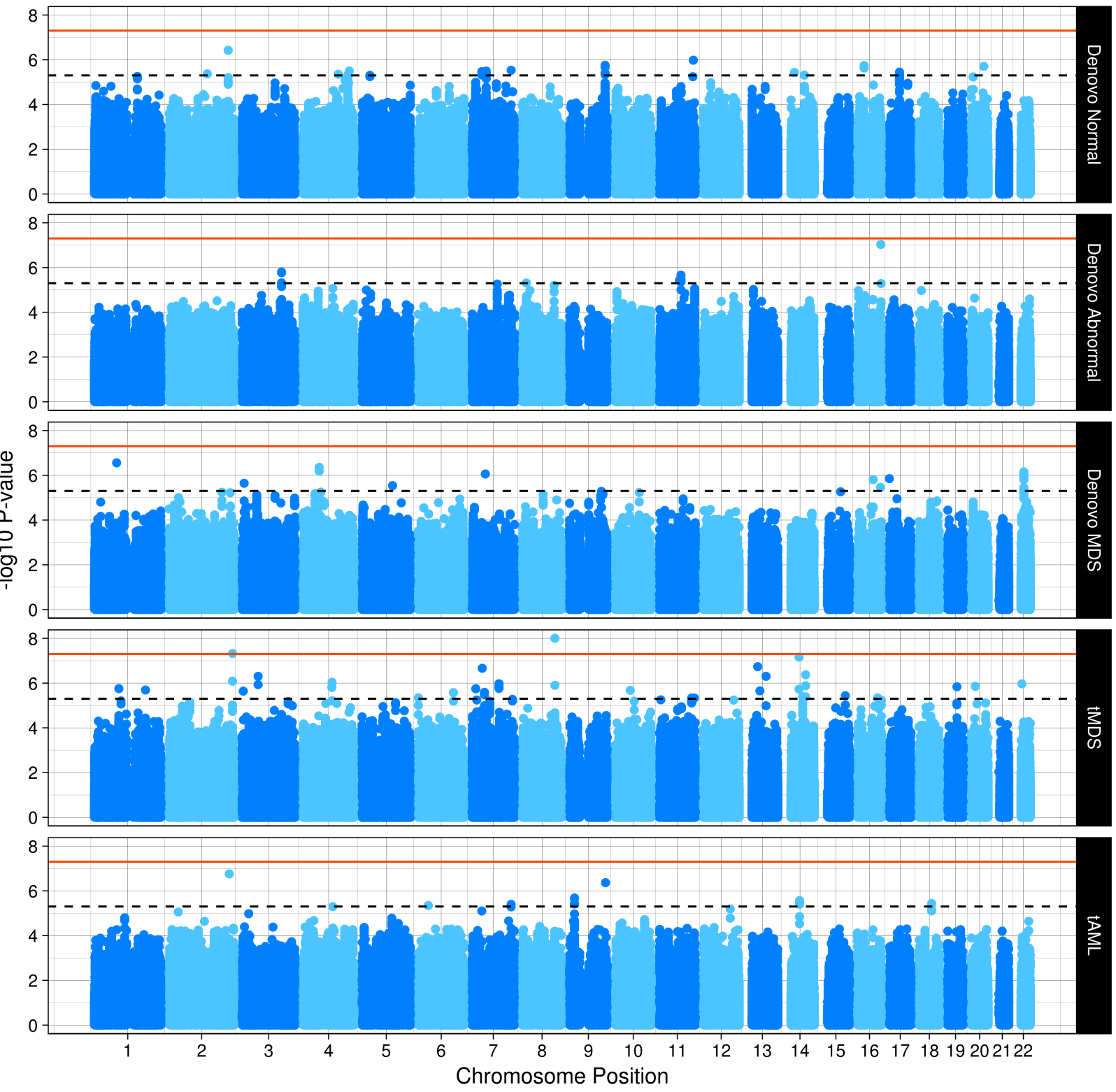

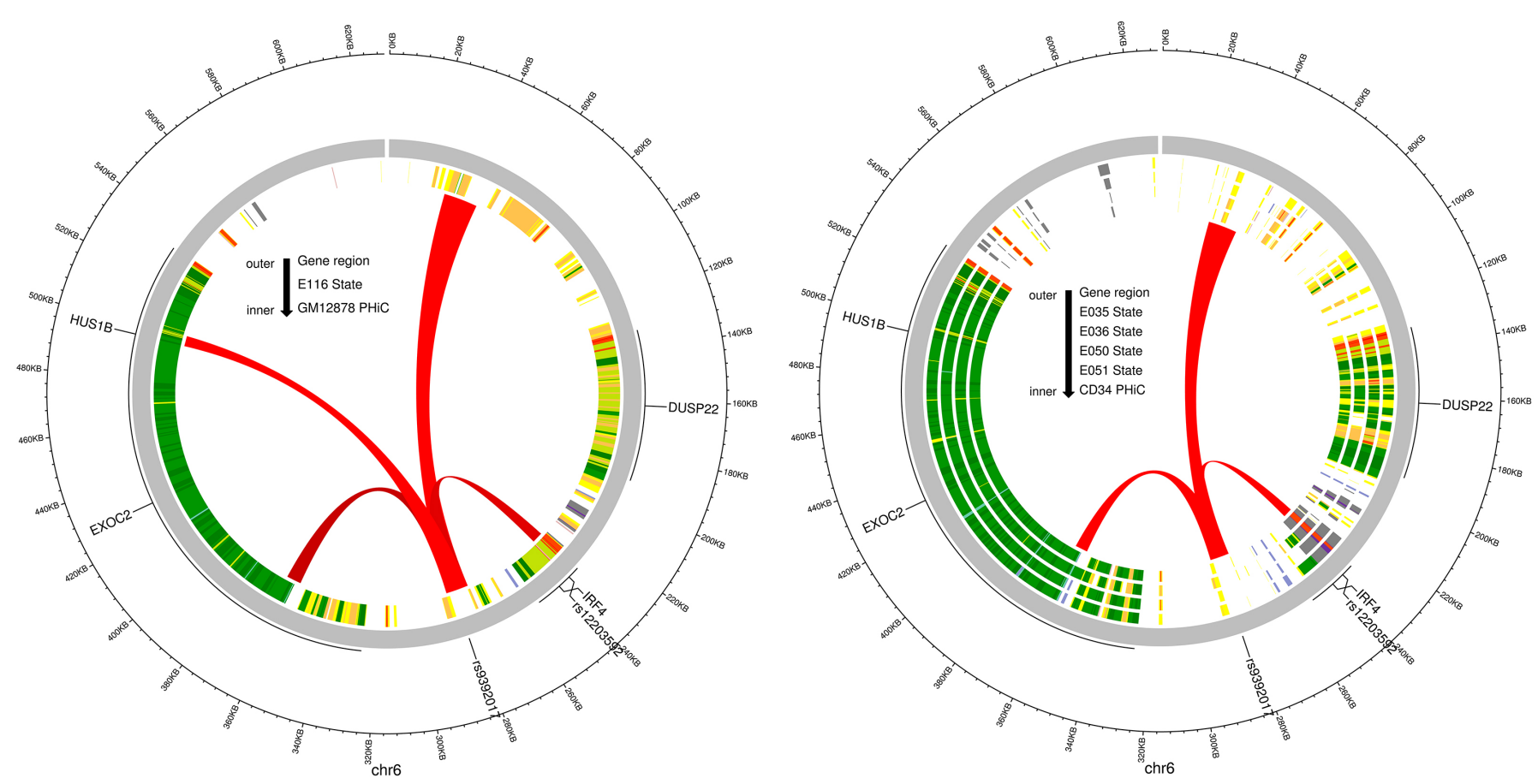

Supplemental Figure 2. Significant chromatin interactions between the promoter region containing AML and MDS susceptibility variant, rs12203592 and the target region containing the previously identified CLL and HL susceptibility variant, rs9392017

The circular plots show significant chromatin interactions between bait-target pairs, defined as a $\mathrm{CHICAGO}$ score $>=5$, designated with red arcs, generated by promoter capture $\mathrm{HI}-\mathrm{C}$ experiments in multiple cell lines. Moving from the outside of the circles inward we see base pair position on chromosome 6 in $\mathrm{Kb}$, protein coding genes are shown in grey (HUS1B, EXOC2, DUSP22 and IRF4), the ENCODE roadmap epigenome chromatin states for (LEFT) E116: lymphoblastoid cell line and the following cell lines (RIGHT) E035:Primary hematopoietic stem cells; E036:Primary hematopoietic stem cells short term culture; E-50:Primary hematopoietic stem cells G-CSF mobilized Female; E-51:Primary hematopoietic stem cells G-CSF mobilized Male.

This figure shows chromatin looping from the reference of the CLL and $\mathrm{HL}$ susceptibility region containing rs9392017 which illustrates this target region interacts with only few adjacent areas and only one transcriptional start site which contains rs12203592 providing support for the role of IRF4 in CLL, HL, AML and MDS. 